\title{
Frequency specific brain networks in Parkinson's disease and comorbid depression
}

\author{
Long Qian $^{1} \cdot$ Yi Zhang ${ }^{2} \cdot$ Li Zheng $^{1} \cdot$ Xuemei Fu $^{1} \cdot$ Weiguo Liu ${ }^{3}$ Y Yuqing Shang ${ }^{4}$. \\ Yaoyu Zhang ${ }^{5,6}$ - Yuanyuan $\mathrm{Xu}^{3} \cdot$ Yijun Liu $^{1} \cdot$ Huaiqiu $\mathrm{Zhu}^{1} \cdot$ Jia-Hong Gao ${ }^{5,6,7}$
}

Published online: 5 February 2016

(C) The Author(s) 2016. This article is published with open access at Springerlink.com

\begin{abstract}
The topological organization underlying the human brain was extensively investigated using resting-state functional magnetic resonance imaging, focusing on a low frequency of signal oscillation from 0.01 to $0.1 \mathrm{~Hz}$. However, the frequency specificities with regard to the topological properties of the brain networks have not been fully revealed. In this study, a novel complementary ensemble empirical mode decomposition (CEEMD) method was used to separate the fMRI time series into five characteristic oscillations with distinct frequencies. Then, the small world properties of brain networks were analyzed for each of these five oscillations in patients $(n=67)$ with depressed Parkinson's disease (DPD, $n$ $=20$ ), non-depressed Parkinson's disease (NDPD, $\mathrm{n}=47$ ) and healthy controls $(\mathrm{HC}, \mathrm{n}=46)$. Compared with $\mathrm{HC}$, the results showed decreased network efficiency in characteristic oscillations from 0.05 to $0.12 \mathrm{~Hz}$ and from 0.02 to $0.05 \mathrm{~Hz}$ for the DPD and NDPD patients, respectively. Furthermore, compared with $\mathrm{HC}$, the most significant inter-group difference across five brain oscillations was found in the basal ganglia
\end{abstract}

Electronic supplementary material The online version of this article (doi:10.1007/s11682-016-9514-9) contains supplementary material, which is available to authorized users.

Yi Zhang

zhangyiuf@gmail.com

$\triangle$ Jia-Hong Gao jgao@pku.edu.cn

1 Department of Biomedical Engineering, Peking University, Beijing 100871, China

2 School of Life Science and Technology, Xidian University, Xi'an, Shaanxi 710071, China

3 Department of Neurology, Affiliated Brain Hospital of Nanjing Medical University, Nanjing 210029, China
( 0.01 to $0.05 \mathrm{~Hz})$ and paralimbic-limbic network (0.02 to 0.22 $\mathrm{Hz}$ ) for the DPD patients, and in the visual cortex (0.02 to 0.05 $\mathrm{Hz}$ ) for the NDPD patients. Compared with NDPD, the DPD patients showed reduced efficiency of nodes in the basal ganglia network $(0.01$ to $0.05 \mathrm{~Hz})$. Our results demonstrated that DPD is characterized by a disrupted topological organization in large-scale brain functional networks. Moreover, the CEEMD analysis suggested a prominent dissociation in the topological organization of brain networks between DPD and NDPD in both space and frequency domains. Our findings indicated that these characteristic oscillatory activities in different functional circuits may contribute to distinct motor and non-motor components of clinical impairments in Parkinson's disease.

Keywords Parkinson's Disease · Depression · Brain Network · Frequency Specificity · Complementary Ensemble Empirical Mode Decomposition

4 Department of Biological Sciences, National University of Singapore, Singapore 119077, Singapore

5 Center for MRI Research, Academy for Advanced Interdisciplinary Studies, Peking University, Beijing 100871, China

6 Beijing City Key Lab for Medical Physics and Engineering, Institute of Heavy Ion Physics, School of Physics, Peking University, Beijing 100871, China

7 McGovern Institute for Brain Research, Peking University, Beijing 100871, China 


\section{Introduction}

Parkinson's disease is the most common movement disorder, characterized by cardinal motor symptoms, including tremor, rigidity, bradykinesia and postural instability (Aarsland et al. 2012), and is associated with various affective symptoms (Luo et al. 2014; F. M. Skidmore et al. 2013). Depression is considered one of the most commonly observed neuropsychiatric disturbances in Parkinson's disease, affecting approximately $35 \%$ of patients with Parkinson's disease (Aarsland et al. 2012). However, the pathophysiology of depression underlying Parkinson's disease remains unclear (McDonald et al. 2003; Postuma et al. 2012; Ravina et al. 2007).

Studies indicate a complex relationship between depression and the motor defect in Parkinson's disease and that these two aspects may influence separate as well as overlapping neural circuits (McDonald et al. 2003; Postuma et al. 2012; Ravina et al. 2007). Resting state functional connectivity (FC) research further demonstrated the association of convergent and divergent brain connectivity patterns between nondepressed Parkinson's disease (NDPD) and depressed Parkinson's disease (DPD) patients (Luo et al. 2014). Whereas NDPD patients are related with abnormalities in the mesolimbic-putamen circuit, the DPD group is associated with aberrations in the prefrontal-limbic network, temporalputamen and mesolimbic-putamen circuits. Based on our knowledge, no work has been reported to delineate the topological organizations of whole-brain functional connectivity networks (FCNs) for both patient groups simultaneously.

In the past decades, graph theoretical analysis has been demonstrated to provide a powerful framework for characterizing topological properties of the brain networks (Bullmore and Sporns 2009), which is typically achieved through all major modalities of magnetic resonance imaging (MRI) and neurophysiological data acquisition from both functional and structural perspectives (Bullmore and Bassett 2011). Under this framework, resting state functional MRI is applied to measure the topological organization of brain networks in this current research. Studies using resting state functional MRI (fMRI) have assessed the potential associations between the topological organization of the brain network and cognitive performance, as well as its association with psychiatric brain disorders (Baggio et al. 2014; Itahashi et al. 2014; Yong Liu et al. 2008; Stam et al. 2007; van den Heuvel et al. 2009).

The majority of previous studies concerning the brain network derived from resting state fMRI focused on a low frequency of signal oscillation from 0.01 to $0.1 \mathrm{~Hz}$ (Bullmore and Sporns 2009; Van Den Heuvel and Hulshoff Pol 2010). However, the frequency specificities concerning the topological properties of the brain networks have not been fully revealed. In addition, previous studies demonstrated motor and non-motor components of clinical impairment in Parkinson's disease contributing to different functional circuit oscillatory activities across multiple frequency bands (Brown 2003; Huebl et al. 2011; Li et al. 2012; Neufeld et al. 1994; Oswal et al. 2013; Sinanovic et al. 2005; Soikkeli et al. 1991). Specifically, motor impairments in Parkinson's disease were associated with a generalized slowing of the electroencephalogram (EEG) and magnetoencephalography (MEG) frequency (Oswal et al. 2013; Soikkeli et al. 1991), an increase of beta frequency waves ( $\mathrm{Li}$ et al. 2012; Oswal et al. 2013), and abnormal subthalamo-pallidal circuit frequency $(<30 \mathrm{~Hz}$ and $>60 \mathrm{~Hz}$ ) (Brown 2003; Oswal et al. 2013); non-motor symptoms in Parkinson's disease such as depression (Huebl et al. 2011) and dementia (Neufeld et al. 1994; Sinanovic et al. 2005) were related to the alterations in alpha and delta rhythms, respectively (Oswal et al. 2013). Nevertheless, the specific BOLD oscillation related to brain networks in NDPD and DPD patients remains to be elucidated.

Derived from the aforementioned literature, it was hypothesized that the functional organizations of brain networks in both patient groups were disrupted. Furthermore, we intended to investigate the association and dissociation in the topological organization of intrinsic brain networks between NDPD and DPD patients from the perspectives of both spatial and spectral spaces. To achieve our goals, a novel data-driven approach named complementary ensemble empirical mode decomposition (CEEMD) was introduced to separate the fMRI time series into several intrinsic mode functions (IMFs) with distinct frequencies. CEEMD can automatically isolate the underlying processes of BOLD activities in a datadriven manner without any assumption of linearity, stationarity, or recourse to any rigid prior chosen band-pass filter, which had been discussed in our previous works (Qian et al. 2015; Song et al. 2014). After applying the CEEMD method, the small world properties of brain networks were analyzed for each of these brain oscillations in both patients and healthy control (HC) groups. Inter-group differences were measured as well.

\section{Materials and methods}

\section{Participants}

The study was approved by Medical Research Ethical Committee of Nanjing Brain Hospital. All patients were recruited from Nanjing Brain Hospital (Nanjing, China) and written informed consents were obtained from all subjects prior to MRI scanning. Seventy patients (37 males) with idiopathic Parkinson's disease and 50 age- and gender- matched healthy controls (23 males, $P>0.05$ ) were recruited. All the subjects were right-handed. The selection of Parkinson's disease patients fulfilled the criteria of UK Parkinson's Disease 
Society Brain Bank for idiopathic Parkinson's disease (Gibb and Lees 1988). All subjects with Mini-Mental State Examination (MMSE) scores $<24$ were also excluded. Patients on dopamine agonists were also excluded, and the dopamine dosing for all patients was stable for at least 4 weeks before and during the study. Laboratory examinations and MRI scans were performed to exclude other diseases. Healthy controls were interviewed to confirm that they had no history of a neurological disorder or psychiatric illness, and no gross abnormality was observed from their brain MRI images (Table 1 and 2).

For each Parkinson's disease patient, all psychometric and neurological evaluations were conducted during a practically defined "on" state. The stage of the disease was evaluated by the Hoehn and Yahr (H\&Y) staging scale (Hoehn and Yahr 1998); motor disability was evaluated using the Unified Parkinson's Disease Rating Scale motor part III (UPDRS III) (Vassar et al. 2012); and global cognitive function was evaluated using the Mini-Mental State Examination (MMSE) score (Folstein et al. 1975). The severity of depression in patients was evaluated by the 17-item Hamilton Depression Rating Scale (HDRS-17) for differentiating NDPD from DPD patients (Stebbins and Goetz 1998; Schrag et al. 2007). All patients with no less than 14 points on the HDRS-17 test were considered depressive (Leentjens et al. 2000). Confirmation of the diagnosis of depression was accomplished by an experienced clinical psychiatrist.

\section{MRI data acquisition}

MR images were acquired on a 3T Siemens Verio system (Siemens, Germany). All subjects were instructed to rest with their eyes closed, not to think about anything in particular, or to fall asleep. A gradient-recalled echo-planar imaging (GREEPI) pulse sequence was used for acquiring resting state functional images, with the parameters as follows: $\mathrm{TR}=2000 \mathrm{~ms}$, $\mathrm{TE}=30 \mathrm{~ms}$, flip angle $=90^{\circ}$; matrix size $=64 \times 64$, FOV $=$ $220 \mathrm{x}^{220 \mathrm{~mm} 2}$, thickness/gap $=3.5 \mathrm{~mm} / 0.6 \mathrm{~mm}$, in-plane resolution of $3.4 \mathrm{~mm} \times 3.4 \mathrm{~mm}$, slices numbers $=31$. The scan for resting state fMRI lasted for 280 seconds, containing 140 brain volumes. Thereafter, anatomical images were acquired using a $\mathrm{T}_{1}$-FLAIR sequence, with the parameters as follows: $\mathrm{TR}=2530 \mathrm{~ms}, \mathrm{TE}=3.34 \mathrm{~ms}$, flip angle $=7^{\circ}$, matrix $=256 \times$ $192, \mathrm{FOV}=256 \mathrm{x}^{256 \mathrm{~mm} 2}$, thickness $/$ gap $=1.33 \mathrm{~mm} / 0.5 \mathrm{~mm}$, slices numbers $=128$.

Table 1 Cortical and subcortical regions of interest defined in the study

\begin{tabular}{|c|c|c|c|c|c|}
\hline Index & Region & Abbr. & Index & Region & Abbr. \\
\hline$(1,2)$ & Precental gyrus & PreCG & $(47,48)$ & Lingual gyrus & LING \\
\hline$(3,4)$ & Superior frontal gyrus, dorsolateral & SFGdor & $(49,50)$ & Superior occipital gyrus & SOG \\
\hline$(5,6)$ & Superior frontal gyrus, orbital part & ORBsup & $(51,52)$ & Middle occipital gyrus & MOG \\
\hline$(7,8)$ & Middle frontal gyrus & MFG & $(53,54)$ & Inferior occipital gyrus & IOG \\
\hline$(9,10)$ & Middle frontal gyrus, orbital part & ORBmid & $(55,56)$ & Fusiform gyrus & FFG \\
\hline$(11,12)$ & Inferior frontal gyrus, opercular part & IFGoperc & $(57,58)$ & Postcentral gyrus & PoCG \\
\hline$(13,14)$ & Inferior frontal gyrus, triangular part & IFGtriang & $(59,60)$ & Superior parietal gyrus & SPG \\
\hline$(15,16)$ & Inferior frontal gyrus, orbital part & ORBinf & $(61,62)$ & Inferior parietal, but supramarginal and angular gyri & IPL \\
\hline$(17,18)$ & Rolandic operculum & ROL & $(63,64)$ & Supramarginal gyrus & SMG \\
\hline$(19,20)$ & Supplementary motor area & SMA & $(65,66)$ & Angular gyrus & ANG \\
\hline$(21,22)$ & Olfactory cortex & OLF & $(67,68)$ & Precuneus & PCUN \\
\hline$(23,24)$ & Superior frontal gyrus, medial & SFGmed & $(69,70)$ & Paracentral lobule & PCL \\
\hline$(25,26)$ & Superior frontal gyrus, medial orbital & ORBsupmed & $(71,72)$ & Caudate nucleus & CAU \\
\hline$(27,28)$ & Gyrus rectus & REC & $(73,74)$ & Lenticular nucleus, putamen & PUT \\
\hline$(29,30)$ & Insula & INS & $(75,76)$ & Lenticular nucleus, pallidum & PAL \\
\hline$(31,32)$ & Anterior cingulate and paracingulate gyri & ACG & $(77,78)$ & Thalamus & THA \\
\hline$(33,34)$ & Median cingulate and paracingulate gyri & DCG & $(79,80)$ & Heschl gyrus & HES \\
\hline$(35,36)$ & Posterior cingulate gyrus & PCG & $(81,82)$ & Superior temporal gyrus & STG \\
\hline$(37,38)$ & Hippocampus & HIP & $(83,84)$ & Temporal pole: superior temporal gyrus & TPOsup \\
\hline$(39,40)$ & Parahippocampal gyrus & PHG & $(85,86)$ & Middle temporal gyrus & MTG \\
\hline$(41,42)$ & Amygdala & AMYG & $(87,88)$ & Temporal pole: middle temporal gyrus & TPOmic \\
\hline$(43,44)$ & Calcarine fissure and surrounding cortex & CAL & $(89,90)$ & Inferior temporal gyrus & ITG \\
\hline$(45,46)$ & Cuneus & CUN & & & \\
\hline
\end{tabular}

The regions are presented according to a prior template obtained from an AAL atlas; odd numbers represent the corresponding brain regions in the left hemisphere, and even numbers denote the specific brain regions in the right hemisphere. AAL: automated anatomical labeling 
Table 2 Demographic and neuropsychological characteristics of all subjects

\begin{tabular}{lllll}
\hline & $\begin{array}{l}\mathrm{HC}(\mathrm{n}=46) \\
\text { Mean } \pm \mathrm{SD}\end{array}$ & $\begin{array}{l}\text { NDPD }(\mathrm{n}=47) \\
\text { Mean } \pm \mathrm{SD}\end{array}$ & $\begin{array}{l}\text { DPD (n=20) } \\
\text { Mean } \pm \text { SD }\end{array}$ & P value \\
\hline Age(years) & $57.74 \pm 5.56$ & $57.64 \pm 7.00$ & $58.05 \pm 7.72$ & $0.973^{*}$ \\
Education(years) & $11.61 \pm 4.95$ & $10.83 \pm 3.29$ & $11.15 \pm 3.12$ & $0.647^{*}$ \\
Gender(M/F) & $22 / 24$ & $25 / 22$ & $9 / 11$ & $0.791 \#$ \\
HDRS-17 & $2.17 \pm 2.42$ & $6.98 \pm 3.29$ & $20.45 \pm 4.58$ & $0.0001^{\mathrm{a}, \mathrm{b}, \mathrm{c}}$ \\
UPDRS III & NA & $26.21 \pm 13.44$ & $27.65 \pm 13.17$ & $0.689^{@}$ \\
H\&Y & NA & $1.63 \pm 0.54$ & $1.43 \pm 0.59$ & $0.175^{@}$ \\
LED(day/mg) & NA & $553.69 \pm 345.43$ & $500.63 \pm 412.41$ & $0.589^{@}$ \\
Duration time of Parkinson's disease & NA & $6.28 \pm 3.35$ & $5.35 \pm 2.81$ & $0.282^{@}$ \\
\hline
\end{tabular}

Values are represented as the mean $\pm \mathrm{SD}$. For comparisons of demographics, *P values are obtained using oneway ANOVA tests; \#P value for the gender distribution in the three groups was obtained using $\chi^{2}$ test. Comparisons of neuropsychological scores among the three groups (HC, NDPD, DPD) were performed using a separate one-way ANOVA. Post hoc pairwise comparisons were performed using $t$-tests. The UPDRS III, H\&Y, LED and Duration time of Parkinson's disease were compared utilizing a two sample t-test between NDPD and DPD for ${ }^{\circledR} \mathrm{P}$ value. $P<0.05$ was considered significant

$N A$ not applicable, $F$ female, $M$ male, $H C$ healthy control, $N D P D$ non-depressed Parkinson's disease, $D P D$ depressed Parkinson's disease, HDRS-17 17-item Hamilton Depression Rating Scale, UPDRS III Unified Parkinson's Disease Rating Scale motor part III, MMSE Mini-Mental State Examination, LED levodopa equivalent dose, $S D$ standard deviation

${ }^{a}$ Post hoc paired comparisons showed significant group differences between HC and NDPD

${ }^{b}$ Post hoc paired comparisons showed significant group differences between HC and DPD

c Post hoc paired comparisons showed significant group differences between NDPD and DPD

\section{Image preprocessing}

Images were analyzed using both FMRIB Software Library (FSL: http://www.fmrib.ox.ac.uk/fsl, version 5.0) and Analysis of Functional NeuroImaging (AFNI: http://afni. nimh.nih.gov/afni, version 2011_12_21_1014). Functional images, after excluding the first 5 time points to ensure signal equilibrium, were corrected for temporal differences and head motions. The mean image was acquired by averaging the volumes. Seven subjects with translation or rotation parameters exceeding $\pm 2 \mathrm{~mm}$ or \pm 2 degrees were excluded, and no inter-group difference was observed with respect to head translation or rotation (both $P>0.05$ ). To enhance the accuracy of normalization, individual structural images were primarily co-registered to the mean functional image, and then we estimated a nonlinear transformation from individual space of the co-registered structural image into MNI152 space. Spatial normalization of the functional image to a standard template (Montreal Neurological Institute) was performed by using the normalization parameters estimated in the last step, resulting in a functional image series of $61 \times 73 \times$ 61 voxels (3-mm isotropic voxels). A regression of nuisance variables from the obtained data, including white matter, ventricular signals, global signals and the six motion parameters determined in the realignment procedure, was performed to reduce the influence of motion and unspecific physiological effects. These images were not spatially smoothed as the previous study suggested (Song et al. 2014). A linear trend was regressed out from the time course of each voxel to remove the signal drifts that arose from scanner instability or other causes.

\section{Empirical mode decomposition}

After preprocessing, CEEMD (Yeh et al. 2010) was performed to separate the time series of each voxel into five intrinsic oscillation rhythms with distinct corresponding frequency bands. CEEMD was originated from empirical mode decomposition (EMD) (Huang et al. 1998) and modified from noise-assisted ensemble empirical mode decomposition (EEMD) (Wu and Huang 2009), which resolved the mode-mixing problem and effectively eliminated the residue noise in each IMF (Wu and Huang 2009; Yeh et al. 2010). The detailed algorithm is presented as follows:

The whole procedure of EMD was depicted by Huang (Huang et al. 1998). Apart from most of the data analysis methods, EMD is an adaptive and efficient method to decompose nonlinear and non-stationary biomedical signals by extracting a series of IMFs from the analyzed signal stage by stage (Lin and Zhu 2012). Mathematically, for a real-valued BOLD signal $\mathrm{x}(\mathrm{t})$, the standard EMD determines a set of $\mathrm{N}$ $\operatorname{IMFs}\left(\operatorname{IMF}_{\mathrm{i}}(\mathrm{t})\right), i=1$ to $N$, and a monotonic residue signal $\mathrm{r}(\mathrm{t})$, so that

$\mathrm{x}(\mathrm{t})=\sum_{\mathrm{i}=1}^{\mathrm{N}} \mathrm{IMF}_{\mathrm{i}}(\mathrm{t})+\mathrm{r}(\mathrm{t})$ 
To ensure that meaningful frequency estimates can be yielded from the time frequency spectra (e.g. no negative frequencies), all the IMFs satisfied the following conditions: the number of zero crossings and the number of extrema equaled or differed at most by one; and the mean value of the upper and lower envelopes defined by the local maxima and local minima was zero at all points. With the above definition of an IMF, all of the signals could be decomposed in the following steps:

1) Identify all of the local extrema;

2) Interpolating all of the minima (resp. maxima) to produce the lower (resp. upper) signal envelope, elow(t) (resp. $\operatorname{eup}(\mathrm{t}))$;

3) To obtain the local mean time course using the following formula:

$\mathrm{m}(\mathrm{t})=[\operatorname{elow}(\mathrm{t})+\operatorname{eup}(\mathrm{t})] / 2$

4) Obtain the "oscillatory mode" from the equation:

$r(t)=x(t)-m(t)$

5) If $r(t)$ meets the standard stopping criterion (shifting process only after the IMF condition is achieved for $\mathrm{S}$ consecutive times, in the current study $\mathrm{S}=3), \mathrm{IMF}_{\mathrm{i}}(\mathrm{t})=\mathrm{r}(\mathrm{t})$ becomes an IMF. Otherwise set $x(t)=r(t)$ and repeat the above steps.

6) To obtain the next IMF, regard the residue $r(t)$ as a new data and repeat the same procedure until $\mathrm{r}(\mathrm{t})$ is smaller than a predetermined value, or $\mathrm{r}(\mathrm{t})$ becames a monotone function. Repeat step 6 for the former case, and terminate the shifting process for the latter case. Thus, a series of IMFs was obtained.

\section{Complementary ensemble empirical mode decomposition}

The method of complementary ensemble empirical mode decomposition (CEEMD) was originated from EMD invented by Huang (Huang et al. 1998) and extended from ensemble empirical mode decomposition (EEMD) by $\mathrm{Wu}(\mathrm{Wu}$ and Huang 2009). The EEMD generates an ensemble of data sets by adding different realizations of white noise with finite amplitude $\varepsilon_{0}$ to the original data. EMD analysis is then applied to each data series of the ensemble; ultimately, the IMFs are achieved by averaging the respective components in each realization over the ensemble (Wu and Huang 2009). The averaging effect of the assisted white noise $\varepsilon_{\mathrm{f}}$ will decrease as:

$\varepsilon_{f}=\varepsilon / \sqrt{\mathrm{NE}}$

In Eq. (4), $\varepsilon=\varepsilon_{0} \operatorname{std}\left(\mathrm{y}_{0}\right), \varepsilon_{0}$ is the input noise level, $\mathrm{y}_{0}$ represents the input signal, and $\mathrm{NE}$ is the ensemble number. Theoretically, NE will approach infinity in order to smooth out the assisted white noise. In practice, $\varepsilon_{0}$ is chosen in the interval of 0.1-0.4; NE of the order of 100 will generally produce satisfactory results and render the residual noise less than a fraction of $1 \%$ of the error (Wu and Huang 2009). In our previous study (Qian et al. 2015), we discussed the selection of parameter $\varepsilon_{0}$. Here, we followed it $\left(\varepsilon_{0}=0.4 ; \mathrm{NE}=100\right)$. To further reduce the final white noise residue in each IMF component and time consumption, a new method, CEEMD, is applied here, where white noise is particularly included in pairs to the original data (i.e. one positive and one negative) to generate two sets of ensemble IMFs (Yeh et al. 2010).

\section{Hilbert weighted frequency}

After the decomposition step, the Hilbert weighted frequency (HWF) of each IMF (Xie and Wang 2006) was applied to reflect the mean oscillation frequency of the IMF in order to visualize the frequency distribution of each IMF in each group (Song et al. 2014). The detailed algorithms were calculated as follows:

Identify all local extrema;

1) For each IMF in Eq. (1), Hilbert transform was applied using the following formula:

$\mathrm{y}_{\mathrm{i}}(\mathrm{t})=\frac{1}{\pi} \mathrm{P} \int \frac{\mathrm{IMF}_{\mathrm{i}}\left(\mathrm{t}^{\prime}\right)}{\mathrm{t}-\mathrm{t}^{\prime}} \mathrm{dt}$

where $\mathrm{P}$ indicates the Caushy principle value.

2) Calculate the corresponding analytical $\operatorname{signal} z_{i}(t)$ :

$z_{i}(t)=\operatorname{IMF}_{i}(t)+i y_{i}(t)=a_{i}(t) e^{-i \theta_{i}(t)}$

Where $a_{i}(t)=\sqrt{\operatorname{IMF}_{i}^{2}(t)+y_{i}^{2}(t)}$ and $\theta_{i}(t)=\arctan$ $\left(\frac{\mathrm{y}_{\mathrm{i}}(\mathrm{t})}{\operatorname{IMF}_{\mathrm{i}}(\mathrm{t})}\right)$

3) The instantaneous frequency of each IMF is defined as:

$w_{i}(t)=\frac{d \theta_{i}(t)}{d t}$

4) The Hilbert weighted frequency (HWF) of each IMF with $\mathrm{m}$ data points is defined as:

$\mathrm{HWF}_{\mathrm{i}}=\frac{\sum_{\mathrm{i}=1}^{\mathrm{m}} \mathrm{w}_{\mathrm{i}}(\mathrm{t}) \mathrm{a}_{\mathrm{i}}^{2}(\mathrm{t})}{\sum_{\mathrm{i}=1}^{\mathrm{m}} \mathrm{a}_{\mathrm{i}}^{2}(\mathrm{t})}$

For most voxels, the decomposition of the time course yielded only four to five IMFs and the frequency range of the first five IMFs covered $0-0.25 \mathrm{~Hz}(\mathrm{TR}=2 \mathrm{~s})$. 
Consequently, only the first five IMFs of each voxel were considered in the current study, denoted as IMF1 to IMF5, and we calculated the HWFs of IMF1 to IMF5 for each voxel to get the histograms of the HWF distribution of IMF1 to IMF5 for the voxels in the whole brain (Fig. 1)

\section{Graph analysis}

\section{Network construction}

In the current study, Pearson's correlation was performed to estimate the IMF dependent correlations between each pair of the 90 cortical and subcortical (90 ROI from the common AAL atlas) BOLD signals derived from each individual set. A set of five $(90 \times 90)$ inter-regional Pearson's correlation matrices was then obtained for each subject. Typical graph

Fig. 1 Histogram of frequency distribution among three groups. The histograms of the HWF distributions display the first five IMFs of the voxels in the whole brain gray matter using the CEEMD approach across all subjects within each group. Colors were assigned in the sequence of red, yellow, blue, magenta and cyan from IMF1 to IMF5. Heights of the histograms represent the amount of voxels with HWF equals the frequency on the horizontal axis. Each of the five histograms from Fig. 1a to

Fig. 1c represents statistics of the whole-brain gray matter voxels within the HC, NDPD and DPD group respectively. The frequency bands denoted by IMFs were similar within each of the three groups. From Fig. 1a to Fig. 1c, the frequency of each IMF fell into a unique frequency band, with the first IMF (IMF1) indicating the highest frequencies (0.12-0.22 Hz), IMF2 from 0.05 to $0.12 \mathrm{~Hz}, \mathrm{IMF} 3$ from 0.02 to $0.05 \mathrm{~Hz}$, IMF4 from 0.01 to 0.03 $\mathrm{Hz}$, and IMF5 being the lowest frequency band from 0 to 0.02 Hz. $H W F$ Hilbert weighted frequency; CEEMD

Complementary Ensemble Empirical Mode Decomposition; $I M F$ Intrinsic mode decomposition; $H C$ healthy control; NDPD non-depressed Parkinson's disease; $D P D$ depressed Parkinson's disease analyses of the weighted networks ignored negative ties while a recent study proposed to incorporate negative weights into analyses of subgraph detection (Power et al. 2011). Here, we followed the traditional approach. False discovery rate (FDR) correction was applied to regulate the expected FDR at a statistical significance threshold of $P<0.05$ across ROI pairs within each subject. Thus, five frequency dependent population-based functional connectivity networks were constructed by capturing the underlying common connectivity pattern of the brain at each IMF for each subject in all groups.

\section{Network analysis}

Small-world analysis Previous studies (He et al. 2008; Liu et al. 2012) demonstrated the two key metrics applied to describe the complex networks in the human brain: clustering
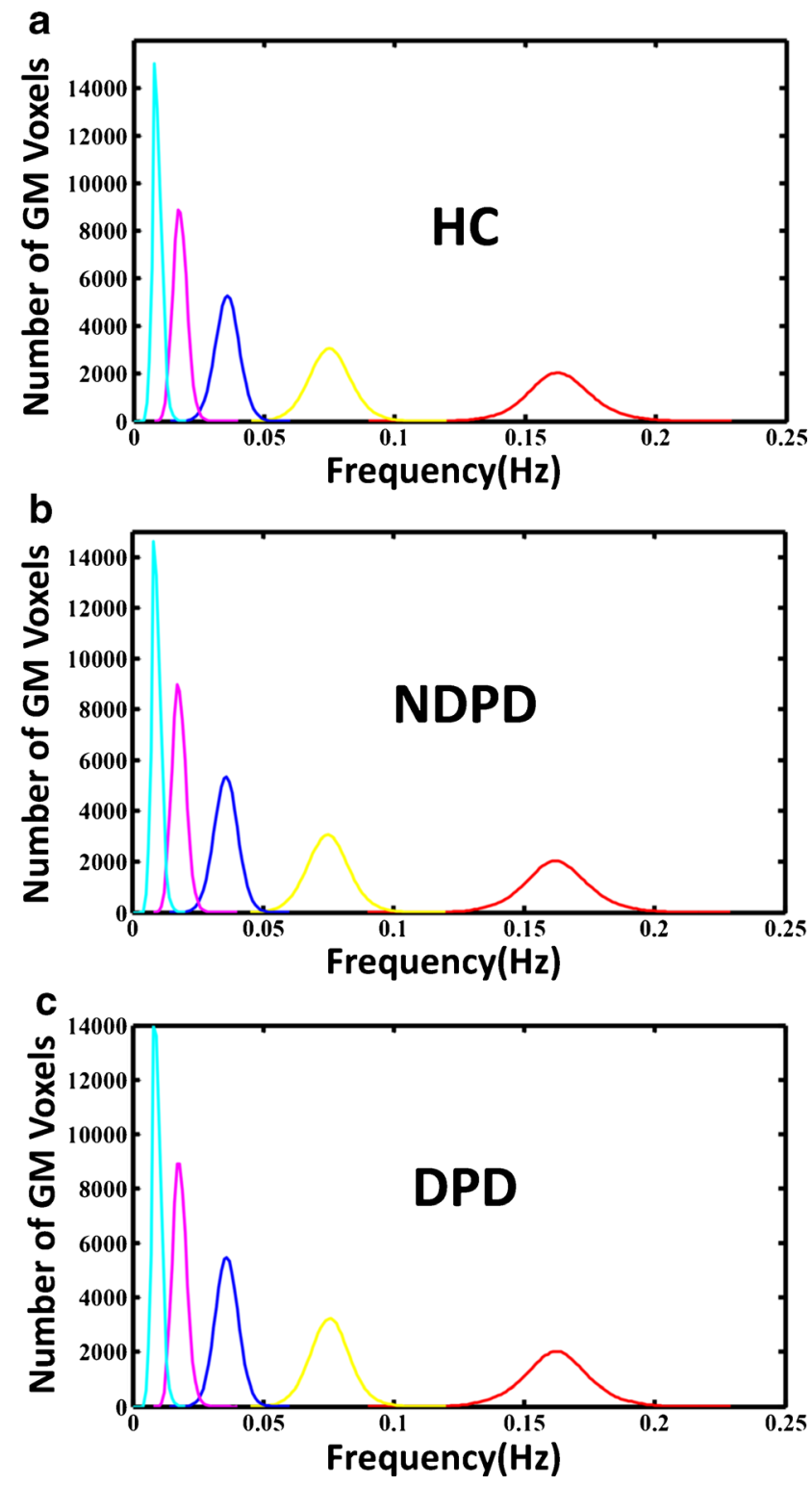
coefficient $(\mathrm{CP})$ and characteristic path length (Lp). In order to investigate the small-world properties, $\mathrm{Cp}$ and Lp were compared with the corresponding random networks (Maslov and Sneppen 2002). A small-world network possessed a significantly higher clustering coefficient value and similar path length than a random network, that is $\gamma=C p($ real $) /$ $C p($ rand $)>1, \lambda=L p($ real $) / L p($ rand $) \approx 1$ (Watts and Strogatz 1998). In order to observe these frequency specificities of brain networks, graph characteristics were calculated at multi-sparsity (or density), which represented the fraction of the present connections to all possible connections (Watts and Strogatz 1998). Notably, in the current study, the estimation of all parameters was under the consideration of a weighted coefficient, which was consistent with a previous study (Zhang et al. 2011). The estimation of all parameters was calculated using the code provided in the Brain Connectivity Toolbox (BCT) (Rubinov and Sporns 2010).

Regional nodal characteristics To determine the nodal (regional) characteristics of frequency specific brain networks, the regional efficiency $\left(E_{i}^{w}\right)$ was computed in the present study, which was defined as the inverse of the mean harmonic shortest path length between the target node and all other nodes in the network (Bai et al. 2012; Liu et al. 2012). Considering the sparsity-dependent regional nodal characteristics, the area under the curve of $E_{i}^{w}$ across a range of the interested sparsity thresholds $\left(\mathrm{S}_{\min }(0.16): 0.01: \mathrm{S}_{\max }(0.35)\right)$ was regarded as the estimation for each node, denoted as $E_{i}^{(w, a u c)}$, where $\mathrm{S}_{\min }$ represented a minimum network sparsity in which all nodes would become fully connected in the five IMF-dependent brain networks (Qian et al. 2015)General speaking, the nodes demonstrating high $E_{i}^{(w, a u c)}$ were considered as the hubs (Hosseini et al. 2012). However, in the current study, to integrate the $E_{i}^{(w, a u c)}$ in each frequency band, nodes were first ranked by their efficiencies in descending order in each frequency band, which resulted in five rank orders for each node. Then, the overall rank order (ORO) for each node was obtained by averaging these five rank orders for the specific node, thereafter, the ORO was normalized by dividing the maximal ORO of the network. Specifically, nodes were considered as hubs if their nodal normalized ORO (NORO) was at least one standard deviation (SD) smaller than the average nodal NORO of the network. Additionally, for reference, hubs defined by the conventional method (Zhang et al. 2011) were also presented in the current study.

\section{Statistical analysis and correlation with clinical variables}

To test the differences in age, education level and neuropsychological scores across the three groups, the data was analyzed using separate one-way ANOVAs. Post-hoc pair wise comparisons were then performed using a $t$-test. The gender difference were also analyzed using a $\chi^{2}$ test. Pair-wise comparisons were performed using a general linear model to determine the inter-group differences in global network measurements and regional efficiency. The effects of age, gender and years of education were adjusted for all of these analyses. A value of $P<0.05$ was considered statistically significant except for when comparing the group effects of regional topological characteristics (statistical significance level at $P<$ 0.01 ). Thus, some nodes may show significant differences in several brain oscillation rhythms between each paired comparisons across the three groups. For simplicity, the maximum inter-group significant difference of one node across five IMFs was reported specifically.

To investigate the clinical relevance of altered brain network regional topology in DPD, the correlations of scores of the 17-item Hamilton Depression Rating Scale (HDRS-17) and nodal efficiency $\left(E_{i}^{(w, a u c)}\right)$ were calculated. Pearson's correlation analysis was applied to control underlying cofounders such as gender, age, and education level $(P<0.05)$.

\section{Results}

\section{Neuropsychological test results}

Twenty patients were diagnosed with DPD according to their HDRS-17 scores. There was no observed inter-group significant difference in age $(\mathrm{F}=0.0275, P=0.973)$, education level $(\mathrm{F}=0.4369, P=0.647)$ or gender $\left(\chi^{2}=0.470, P=0.791\right)$. The HDRS-17 scores varied significantly across the three groups ( $\mathrm{F}$ $=248.16, P<0.0001)$. No significant difference existed in UPDRS III $(\mathrm{t}=-0.4029, P=0.6884), \mathrm{H} \& \mathrm{Y}(\mathrm{t}=1.3729, P=$ $0.1745), \operatorname{LED}(\mathrm{t}=0.5429, P=0.5890)$ or Parkinson's disease duration $(\mathrm{t}=1.0843, P=0.2822)$ between NDPD and DPD groups, as presented in Table 2. The inter-gender difference for HDRS-17 scores was not statistically significant in either the NDPD group (male: $6.92 \pm 3.417$; female: $6.62 \pm 2.599 ; P=$ 0.757 ) or the DPD group (male: $20.56 \pm 6.044$; female: $20.36 \pm$ 3.264; $P=0.933$ ), and depression severity was not correlated with age in the NDPD group $(\mathrm{R}=0.085, P=0.051)$.

\section{Frequency properties of IMF in NDPD, DPD and HC}

The histogram of HWF distribution presented in Fig. 1 demonstrated the first five IMFs (IMFs, $s=1,2,3,4$ or 5) of voxels determined using the CEEMD method in the whole brain grey matter across all subjects within each groups. The result showed that the same IMF derived from all voxels fell approximately into the same frequency band, where the five IMF components covered a frequency band ranging from 0 to $0.22 \mathrm{~Hz}$ [27]. Moreover, the frequency bands denoted by IMFs were similar across the three groups. As demonstrated from Fig. 1a to Fig. 1c, 
the first IMF (IMF1) indicated the highest frequencies of 0.12$0.22 \mathrm{~Hz}, \mathrm{IMF} 2$ of 0.05 to $0.12 \mathrm{~Hz}, \mathrm{IMF} 3$ of 0.02 to $0.05 \mathrm{~Hz}$, IMF4 of 0.01 to $0.03 \mathrm{~Hz}$, and IMF5 of 0 to $0.02 \mathrm{~Hz}$ as the lowest frequency band. The results suggested that CEEMD could adaptively decompose the original time series into several similar intrinsic oscillatory modes within distinct frequency bands across the three groups.

\section{Frequency specific global topology alterations in NDPD and DPD}

The significant inter-group global topological difference was observed in IMF1, IMF2 and IMF3 components (Fig. 2), but not in other frequency bands. In addition, all groups showed a small-world organization of these five frequency specific FNCs (Fig. 2).
Fig. 2 shows the mean values of $\mathrm{Cp}, \mathrm{Lp}, \gamma$ and $\lambda$ as a function of sparsity for all groups, respectively. The network metrics were calculated for each individual frequency specific FC graph, while the group mean values and significantly different levels were displayed.

Within IMF1, a significant inter-group difference of $\gamma$ was observed at a narrow range of sparsity (from 0.1 to 0.12 ). Within the IMF2, a significantly higher Lp was observed in the DPD group over a wide range of densities as compared to HC (Fig. 2). Both the NDPD and DPD groups showed a significantly lower $\lambda$ at a range of the density threshold from 0.1 to 0.35 . The same network parameters were compared and presented within IMF3 in Fig. 2. Among which, a significantly lower $\gamma$ and higher Lp were observed in the NDPD group over a wide range of densities relative to $\mathrm{HC}$. Both the NDPD and DPD groups further
IMF1
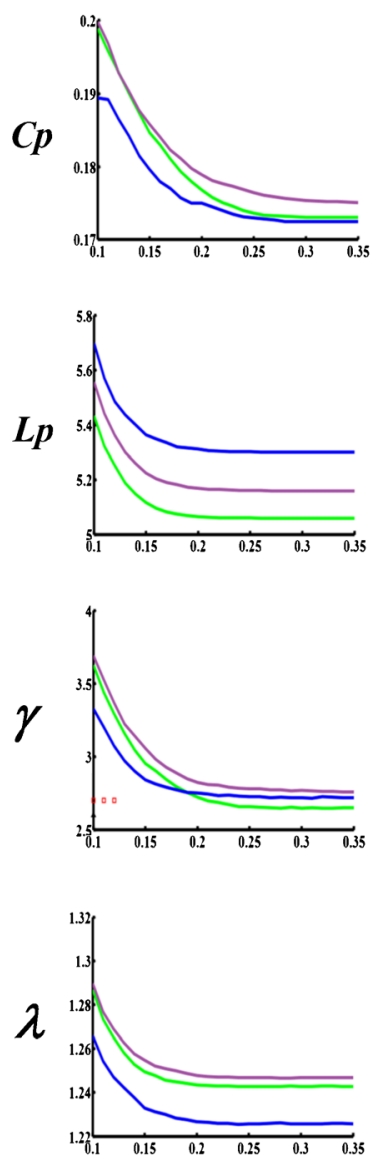

IMF2
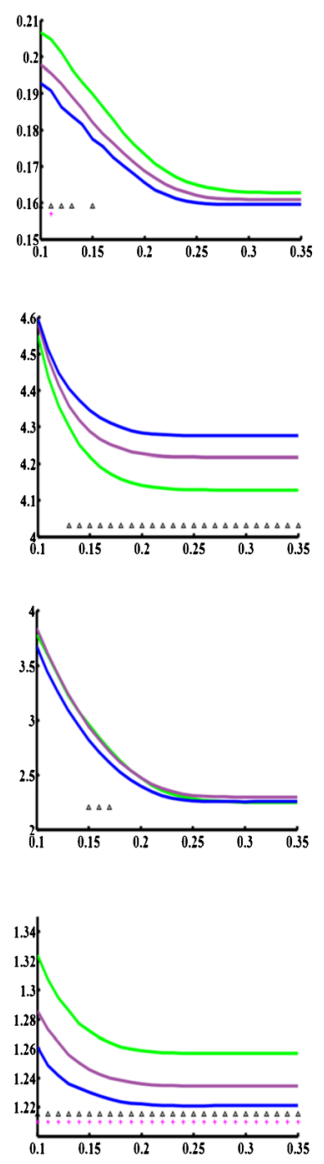

IMF3
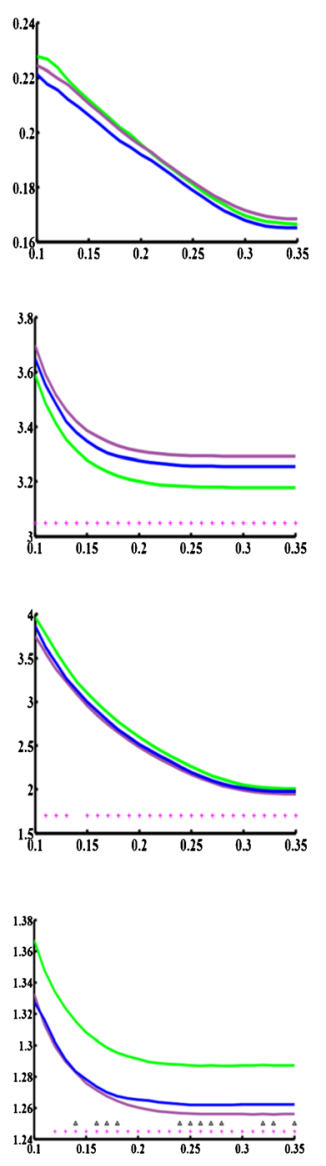

IMF4
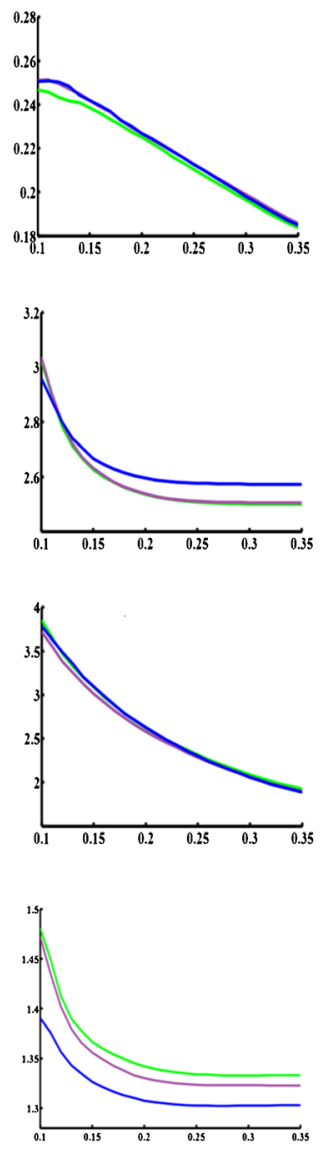

IMF5
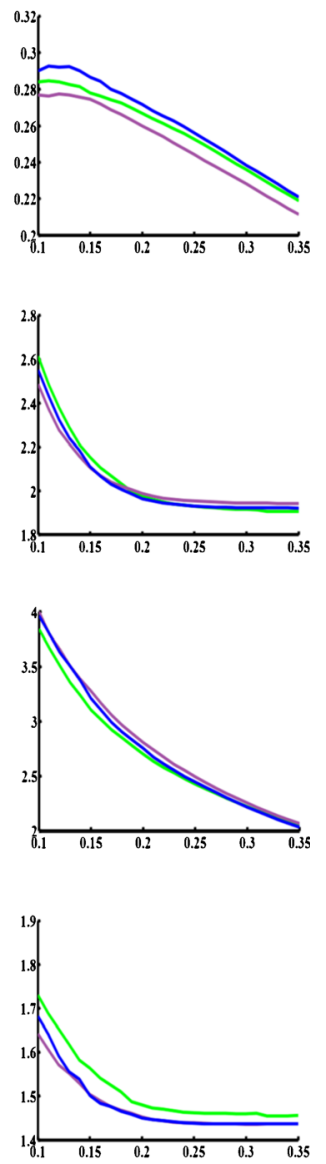

\section{Sparsity}

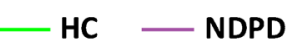

Fig. 2 Global measures of frequency specific brain networks were quantified in the HC, NDPD and DPD patients. All groups showed a small-world organization of these five frequency specific FNCs. The significant inter-group overall global topological differences are presented in IMF2 and IMF3. The data points are marked with a red plus sign to indicate a significant difference (HC vs. NDPD; $P<0.05$ ), and with a black asterisk to indicate a significant difference (HC vs. DPD; $P<0.05) . I M F$ Intrinsic mode decomposition; $H C$ healthy control; $N D P D$ non-depressed Parkinson's disease; $D P D$ depressed Parkinson's disease 
showed a significantly lower $\lambda$ over a wide range of densities compared with HC.

\section{Nodal characteristics}

\section{Identification of network hubs}

Fig. 3 and Table 3 show the nodes identified as hubs. In line with previous reports (Achard et al. 2006; Zhang et al. 2011; Nijhuis et al. 2013; Tomasi and Volkow 2011), several common nodes were observed to present the network hub property across the three groups. In particular, seven of the hub regions were identified for all groups including bilateral medial orbital parts of superior frontal gyrus (ORBsupmed), rectus gyrus (REC), superior temporal gyrus (STG) and right temporal pole part of superior temporal gyrus (TPOsup). Four hub regions: bilateral medial parts of superior frontal gyrus (SFGmed), left TPOsup and right insula (INS), were identified as hub regions in both patient groups, but not in the HC group. Instead of the NDPD group, hub regions in the occipital lobe were only observed in HC and DPD groups - those were the left superior occipital gyrus (SOG) and lingual gyrus (LING) in both the
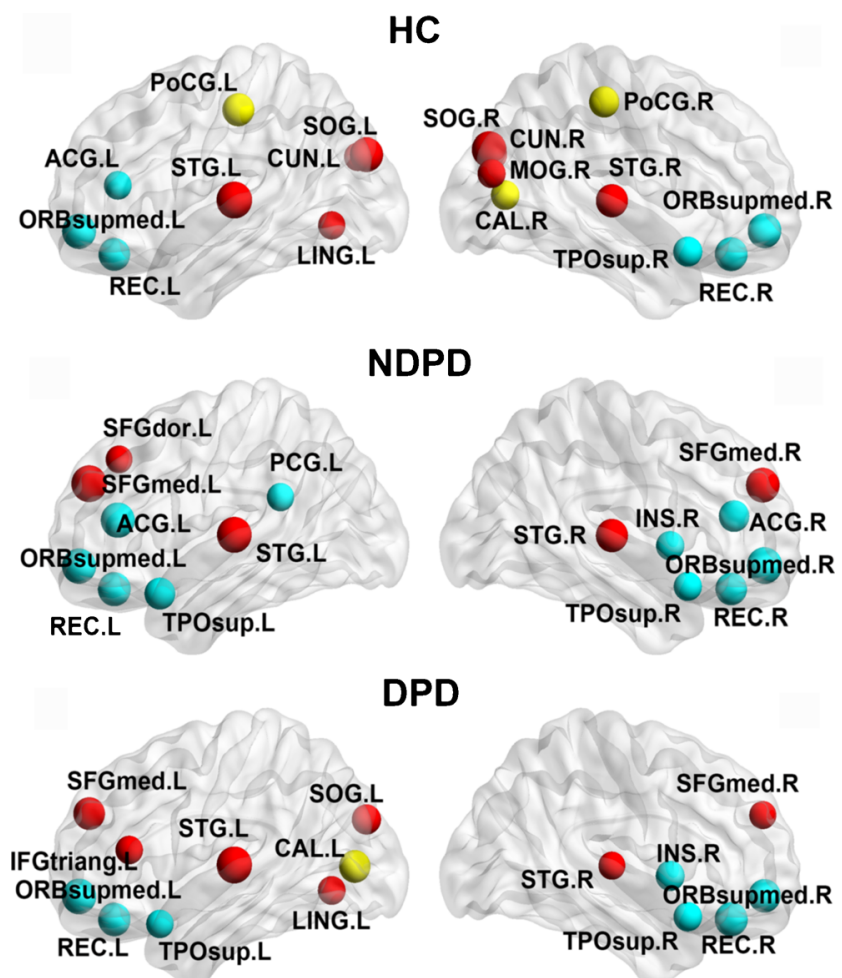

Fig. 3 The distribution of hub regions in the HC, NDPD and DPD groups. Hub regions were visualized using BrainNet view (NKLCNL, Beijing Normal University). Three-dimensional rendering maps showed the hub regions defined by normalized overall rank order for each node (Table 3). The hub nodes are colored in red, yellow, and cyan indicating Associations, Primary, Paralimbic regions, respectively. The abbreviations of the regions are shown in Table 1. HC healthy control; NDPD non-depressed Parkinson's disease; $D P D$ depressed Parkinson's disease
Table 3 Hub regions in HC, NDPD and DPD groups

\begin{tabular}{|c|c|c|c|}
\hline & Hub regions & Functional classification & Normalized ORO \\
\hline \multirow[t]{17}{*}{$\mathrm{HC}$} & STG.L & Association & 0.0473 \\
\hline & SOG.R & Association & 0.0709 \\
\hline & ORBsupmed.L & Paralimbic & 0.0898 \\
\hline & ORBsupmed.R & Paralimbic & 0.0993 \\
\hline & SOG.L & Association & 0.1064 \\
\hline & REC.R & Paralimbic & 0.1229 \\
\hline & PoCG.L & Primary & 0.1418 \\
\hline & STG.R & Association & 0.1418 \\
\hline & REC.L & Paralimbic & 0.1489 \\
\hline & PoCG.R & Primary & 0.1915 \\
\hline & CUN.R & Association & 0.2080 \\
\hline & TPOsup.R & Paralimbic & 0.2222 \\
\hline & CAL.R & Primary & 0.2364 \\
\hline & MOG.R & Association & 0.2506 \\
\hline & ACG.L & Paralimbic & 0.2577 \\
\hline & CUN.L & Association & 0.2600 \\
\hline & LING.L & Association & 0.2695 \\
\hline \multirow[t]{15}{*}{ NDPD } & SFGmed.L & Association & 0.0437 \\
\hline & ORBsupmed.R & Paralimbic & 0.0506 \\
\hline & STG.L & Association & 0.0713 \\
\hline & ORBsupmed.L & Paralimbic & 0.0851 \\
\hline & ACG.L & Paralimbic & 0.0874 \\
\hline & SFGmed.R & Association & 0.0989 \\
\hline & REC.L & Paralimbic & 0.1195 \\
\hline & STG.R & Association & 0.1379 \\
\hline & REC.R & Paralimbic & 0.1540 \\
\hline & TPOsup.L & Paralimbic & 0.1747 \\
\hline & ACG.R & Paralimbic & 0.1816 \\
\hline & INS.R & Paralimbic & 0.2391 \\
\hline & TPOsup.R & Paralimbic & 0.2460 \\
\hline & PCG.L & Paralimbic & 0.2667 \\
\hline & SFGdor.L & Association & 0.2782 \\
\hline \multirow[t]{15}{*}{ DPD } & STG.L & Association & 0.0566 \\
\hline & ORBsupmed.L & Paralimbic & 0.0792 \\
\hline & REC.R & Paralimbic & 0.1154 \\
\hline & REC.L & Paralimbic & 0.1312 \\
\hline & ORBsupmed.R & Paralimbic & 0.1335 \\
\hline & SFGmed.L & Association & 0.1493 \\
\hline & CAL.L & Primary & 0.1629 \\
\hline & SOG.L & Association & 0.2172 \\
\hline & INS.R & Paralimbic & 0.2240 \\
\hline & TPOsup.R & Paralimbic & 0.2376 \\
\hline & LING.L & Association & 0.2557 \\
\hline & IFGtriang.L & Association & 0.2624 \\
\hline & TPOsup.L & Paralimbic & 0.2647 \\
\hline & SFGmed.R & Association & 0.2738 \\
\hline & STG.R & Association & 0.2828 \\
\hline
\end{tabular}

Major "hubs" of the brain networks in each group defined by normalized overall rank order. The cortical regions were classified as primary, association, and paralimbic. ORO overall rank order. For the abbreviations of the regions, refer to Table 1 
$\mathrm{HC}$ and DPD groups, and the bilateral cuneus (CUN), right SOG, calcarine fissure (CAL) and middle occipital gyrus (MOG) in the HC group, and left CAL in the DPD group. Bilateral postcentral gyri (PoCG) were identified as hub regions in the $\mathrm{HC}$ group, but not in the other two groups. Hubs in the cingulate gyrus were demonstrated in the $\mathrm{HC}$ and NDPD groups - left anterior cingulate gyrus (ACG) in both $\mathrm{HC}$ and NDPD groups, left posterior cingulate gyrus (PCG) and right ACG in the NDPD group - but no hub in the cingulate gyrus for the DPD group. In addition, the left dorsal superior frontal gyrus (SFGdor) and triangular part of inferior frontal gyrus (IFGtriang) were identified as a hub in the NDPD and DPD group, respectively. Moreover, convergent results between the hubs were defined by both NORO (Fig. 3 and Table 3) and the conventional method (Supplementary Fig. 1, Supplementary Tables 1 to 5) (Zhang et al. 2011). The hubs identified in the NDPD group were predominantly detected in regions of paralimbic cortices. Similar findings have been reported in a previous study of temporal lobe epilepsy, where the authors also observed a higher proportion of paralimbic hubs in patients than in controls (Zhang et al. 2011).

\section{Between-group difference in regional efficiency}

Along with the discovery of a disrupted frequency-specific global network organization, pair-wise group comparisons on regional efficiency $\left(E_{i}^{w}\right)$ in distinct frequency bands revealed the alterations of nodal efficiency in both patient groups (Fig. 4).

Compared to HC, the regions with the most significant group effects were mainly concentrated in IMF3 and predominantly observed in the visual cortex in the NDPD group (Fig. 4a). Decreased $E_{i}^{w}$ of IMF3 in the NDPD group was observed in the bilateral lingual gyri (LING), superior occipital gyrus (SOG), left postcentral gyri (PoCG), right calcarine fissure (CAL), cuneus (CUN), middle occipital gyrus (MOG), fusiform gyrus (FFG) and angular gyrus (ANG), while increased $E_{i}^{w}$ of component IMF4 was observed in the left supplementary motor area (SMA) (Fig. 4a and Table 4).

Relative to $\mathrm{HC}$, the most significant observed group effects mainly focused on IMF2 and IMF3, and were predominately distributed in the paralimbic-limbic system in the DPD group (Fig. 4b). Decreased $E_{i}^{w}$ was observed in DPD patients in the left orbital part of superior frontal gyrus (ORBsup) from IMF1; bilateral amygdala (AMYG), left orbital part of middle frontal gyrus (ORBmid), hippocampus (HIP), and right precentral gyrus (PreCG) from IMF2; bilateral caudate nucleus (CAU), left anterior cingulate gyrus (ACG) and PoCG from IMF3; and right SOG from IMF4. Meanwhile, increased $E_{i}^{w}$ of component IMF5 was observed in the left insula (INS) (Fig. $4 \mathrm{~b}$ and Table 4). Compared with NDPD, the most obvious observation for a HC vs NDPD

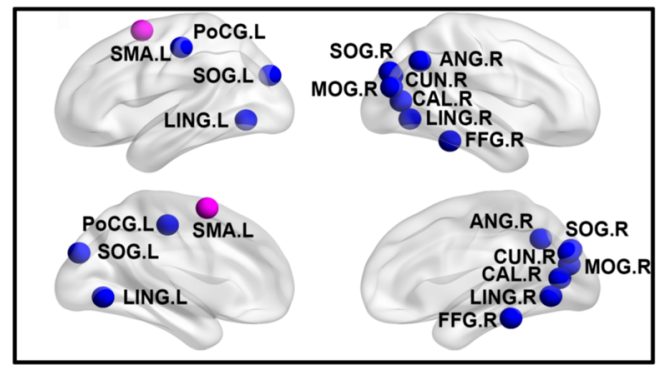

\section{b HC vs DPD}
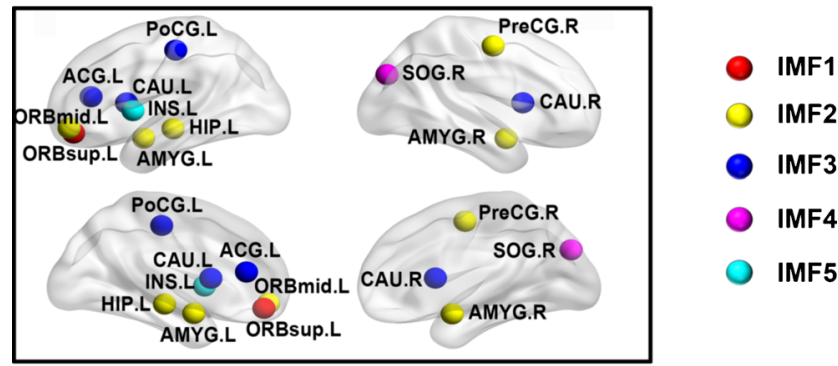

\section{DPD vs NDPD}

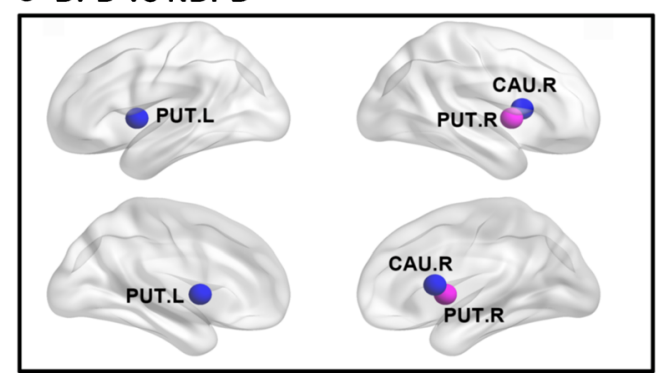

Fig. 4 The distribution of brain regions with most significant differences in nodal efficiency among the HC, NDPD and DPD groups across five frequency bands. Nodes from Fig. 4a to Fig. 4c represent the brain regions with the most significant differences across the three groups in the regional efficiencies across five identical frequency bands $(P<0.01$, uncorrected). Colors were assigned in the sequence of red, yellow, blue, magenta and cyan from IMF1 to IMF5. The nodes with significant topological alterations were mainly distributed in the visual cortex in NDPD patients, and in the paralimbic-limbic and basal ganglia networks in DPD patients. The significant disrupted nodal topological characteristic was dominated in the frequency bands from 0.02 to $0.05 \mathrm{~Hz}$ in the visual cortex in the NDPD group, as well as from 0.01 to $0.05 \mathrm{~Hz}$ in basal ganglia and from 0.02 to $0.22 \mathrm{~Hz}$ in the paralimbic-limbic network in the DPD group. Brain regions were visualized using the BrainNet viewer (NKLCNL, Beijing Normal University). For the abbreviations of the regions, refer to Table 1. IMF Intrinsic mode decomposition; $H C$ healthy control; NDPD non-depressed Parkinson's disease; DPD depressed Parkinson's disease

group effects was concentrated in IMF3 and IMF4 components and was distributed in the subcortical network in the DPD group (Fig. 4c). Decreased $E_{i}^{w}$ was observed in the right CAU and left putamen (PUT) from IMF3, and right PUT from IMF4 in the DPD group (Fig. 4c and Table 4). In addition, $E_{i}^{w}$ of the right $\mathrm{CAU}(\mathrm{R}=-0.3366, P=0.0054)$ in $\mathrm{IMF} 3$ and right PUT (R $=-0.3558, P=0.0031)$ in IMF4 were negatively correlated with the HDRS-17 score (Fig. 5). 
Table 4 Between-group differences of nodal efficiency among NDPD, DPD and HC

\begin{tabular}{|c|c|c|c|c|c|}
\hline & & Regions & $\begin{array}{l}\text { Functional } \\
\text { classification }\end{array}$ & $\begin{array}{l}\text { Anatomical } \\
\text { classification }\end{array}$ & $\begin{array}{l}\text { Difference values } \\
\text { (P values) }\end{array}$ \\
\hline \multirow[t]{14}{*}{ HC vs NDPD } & IMF1 & NS & NS & NS & NS \\
\hline & IMF2 & NS & NS & NS & NS \\
\hline & IMF3 & CAL.R & Primary & Occipital & $3.09(0.0027)$ \\
\hline & & CUN.R & Association & Occipital & $2.92(0.0044)$ \\
\hline & & LING.L & Association & Occipital & $2.72(0.0079)$ \\
\hline & & LING.R & Association & Occipital & $3.14(0.0023)$ \\
\hline & & SOG.L & Association & Occipital & $3.16(0.0022)$ \\
\hline & & SOG.R & Association & Occipital & $3.25(0.0016)$ \\
\hline & & MOG.R & Association & Occipital & $4.34(0.00004)$ \\
\hline & & FFG.R & Association & Temporal & $3.00(0.0035)$ \\
\hline & & PoCG.L & Primary & Parietal & $2.71(0.0081)$ \\
\hline & & ANG.R & Association & Parietal & $2.94(0.0041)$ \\
\hline & IMF4 & SMA.L & Association & Frontal & $-3.42(0.0009)$ \\
\hline & IMF5 & NS & NS & NS & NS \\
\hline \multirow[t]{12}{*}{ HC vs DPD } & IMF1 & ORBsup.L & Paralimbic & Prefontal & $2.80(0.0071)$ \\
\hline & IMF2 & PreCG.R & Primary & Frontal & $3.18(0.0023)$ \\
\hline & & ORBmid.L & Paralimbic & Prefontal & $2.70(0.0090)$ \\
\hline & & HIP.L & Limbic & Temporal & $2.72(0.0084)$ \\
\hline & & AMYG.R & Subcortical & Temporal & $2.85(0.0058)$ \\
\hline & & AMYG.L & Subcortical & Temporal & $2.79(0.0069)$ \\
\hline & IMF3 & ACG.L & Paralimbic & Prefontal & $3.10(0.0029)$ \\
\hline & & PoCG.L & Primary & Parietal & $2.74(0.0080)$ \\
\hline & & CAU.L & Subcortical & Subcortical & $4.39(0.00004)$ \\
\hline & & CAU.R & Subcortical & Subcortical & $2.94(0.0046)$ \\
\hline & IMF4 & SOG.R & Association & Occipital & $2.94(0.0045)$ \\
\hline & IMF5 & INS.L & Paralimbic & Subcortical & $-2.77(0.0073)$ \\
\hline \multirow[t]{6}{*}{ DPD vs NDPD } & IMF1 & NS & NS & NS & NS \\
\hline & IMF2 & NS & NS & NS & NS \\
\hline & IMF3 & CAU.R & Subcortical & Subcortical & $-2.99(0.0039)$ \\
\hline & & PUT.L & Subcortical & Subcortical & $-2.68(0.0093)$ \\
\hline & IMF4 & PUT.R & Subcortical & Subcortical & $-3.15(0.0025)$ \\
\hline & IMF5 & NS & NS & NS & NS \\
\hline
\end{tabular}

The functional connectivity networks for each participant in five specific brain oscillations were constructed using an AAL template. Pair wise comparisons were performed using a general linear model (adjusted for the effects of age, gender and years of education) to determine the inter-group differences for regional efficiency. A value of $P<0.01$ (uncorrected) was considered statistically significant. Only the maximum inter-group significant difference of regional efficiency in one IMF was reported specifically. NS: Not significant. For difference values, negative values in each pair-wise comparison represent $\mathrm{HC}<\mathrm{NDPD}, \mathrm{HC}<\mathrm{DPD}$, and DPD $<$ NDPD respectively. $I M F$ Intrinsic mode decomposition; $H C$ healthy control; $N D P D$ non-depressed Parkinson's disease; $D P D$ depressed Parkinson's disease; the abbreviations of the regions, refer to Table 1

\section{Discussion}

In this current study with the utilization of resting state fMRI, the results suggested the dissociations of topological organizations of brain networks between NDPD and DPD patients in both spatial and spectral domains. Our data revealed three major findings: i) Compared to $\mathrm{HC}$, the increased characteristic path length was exclusively observed in IMF2 in the DPD group and in IMF3 in the NDPD group; (ii) The nodes with significant topological alterations were mainly distributed in the visual cortex in NDPD patients, or in the paralimbiclimbic and basal ganglia networks in DPD patients at the nodal topological level; (iii) The significant disrupted nodal topological characteristic was dominated in the frequency band from 0.02 to $0.05 \mathrm{~Hz}$ in the visual cortex in the NDPD group, as well as from 0.01 to $0.05 \mathrm{~Hz}$ in the basal ganglia and from 0.02 to $0.22 \mathrm{~Hz}$ in the paralimbic-limbic network in the DPD group. 
Right Caudate(IMF3)

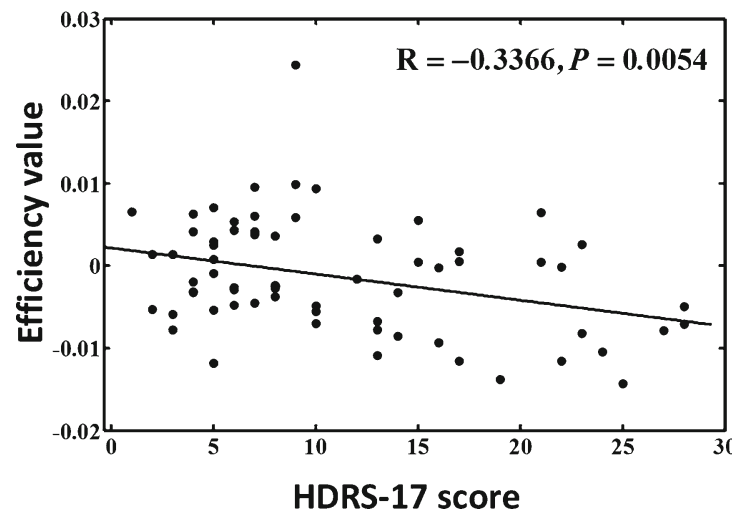

Fig. 5 Relationship between frequency specific nodal efficiency and clinical variables. The results of the correlation analysis between the HDRS-17 score ( $x$-axis) and frequency specific nodal efficiency in the left thalamus $(\mathrm{R}=-0.2721, P=0.0259)$ and right caudate nucleus $(\mathrm{R}=$ -

Global network measurements (i.e. $\gamma$ and $\lambda$ ) indicated a small-world organization of all frequency specific brain networks in all groups. Compared to HC, the increased characteristic path length observed in IMF2 and IMF3 of the DPD and NDPD groups respectively, indicated an altered organization of the brain networks with specific brain oscillation leading to lower efficiency. Additionally, a previous electrophysiological study suggested that distinct motor and non-motor components of clinical impairment in Parkinson's disease might be detected as oscillatory activities across multiple frequency bands and their cross-frequency interactions within spatially segregated loops of the basal ganglia-thalamocortical system (Oswal et al. 2013). Performing resting state fMRI, dissociation in global topological patterns of brain networks between NDPD and DPD patients in the frequency domain was observed. The results indicated the potential disassociation of global FC differences with their corresponding activities in distinct resting oscillation rhythms. In addition, the results of some topological profiles, including Cp in IMF2, and $\gamma$ in IMF1 and IMF2, were difficult to interpret due to the fact that their inter-group differences were only significant at a narrow range of sparsity. Future studies may classify this topic in a large cohort.

Regarding regional properties, brain regions with reduced efficiency in NDPD were predominately located in the occipital cortex, which was consistent with previous studies (Gottlich et al. 2013; Luo et al. 2014; Skidmore et al. 2011). Previous studies documented that the Parkinson's disease-related visual damages ranged from deficits in basic perceptual and semantic visual processing at an early stage of cognitive deterioration (Laatu et al. 2004; Cardoso et al. 2010), problems in motion and orientation discrimination (Bodis-Wollner and Paulus 1999; Trick et al. 1994) to even visual hallucinations (Barnes and David 2001; Mindham 2001). The observed modification of nodal efficiency in the right ANG might account for the non-
Right Putamen(IMF4)

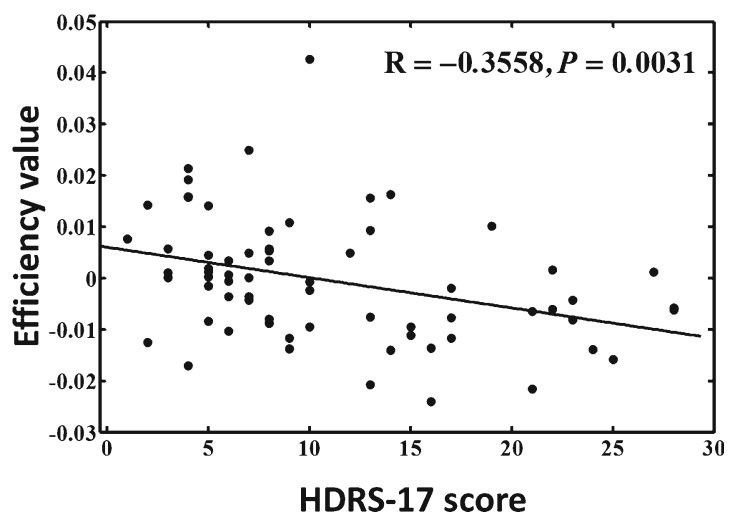

$0.3366, P=0.0054)$ in IMF3 component, and of the right putamen $(\mathrm{R}=$ $0.3558, P=0.0031)$ and pallidum $(\mathrm{R}=-0.3184, P=0.0086)$ in IMF4. HDRS-17 17-item Hamilton Depression Rating Scale; IMF Intrinsic mode decomposition

motor symptoms of patients with Parkinson's disease such as linguistic deficits and executive dysfunction (Altmann and Troche 2011; Kudlicka et al. 2011; Murray and Rutledge 2014; Shirer et al. 2012). Meanwhile, decreased efficiency in the left PoCG might be associated with the motor symptoms in Parkinson's disease, which was consistent with a previous study (Sharman et al. 2013). Similarly, the disrupted BOLD signal activities in the PoCG and PreCG were also observed in the DPD group. On the other hand, a higher efficiency observed in the left supplementary motor area might reflect the compensatory mechanisms in Parkinson's disease (Kwak et al. 2010; Yu et al. 2013; Sharman et al. 2013).

The occurrence of depression in Parkinson's disease might arise from the disturbance in the paralimbic-limbic system as well as the basal ganglia network. Our results highlighted the critical role of nodes in the paralimbic-limbic system in DPD, which was consistent with previous studies (Luo et al. 2014; Skidmore et al. 2013). In addition, extensive evidence indicated that the abnormal function of the striatum and the associated limbic-basal ganglia circuitry played a role in the emotional processing system in patients with depression (Jiao et al. 2011; Lui et al. 2011; Marchand et al. 2012). Moreover, a recent study reported by Joutsa et al. (2013) suggested that impaired striatal dopaminergic function was related to depression symptoms in Parkinson's disease. In the current study, decreased nodal efficiency observed in DPD patients in bilateral CAU (compared with HC) as well as bilateral PUT and right CAU (compared with NDPD) further supported the hypothesis. Additionally, the increased regional efficiencies in the left INS observed in DPD patients might have resulted from depression symptoms in Parkinson's disease. For instance, recent studies (Connolly et al. 2013; Frodl et al. 2010) indicated an increased FC between the subgenual anterior cingulate and insula cortex in patients with depression, which goes along with our findings that the subgenual anterior 
cingulate was likely to be the vital hubs in the neural circuits associated with depression in Parkinson's disease. It was disputable whether regional efficiency differences demonstrated by fMRI in the high frequency band (IMF1, 0.12 0.22 Hz) were attributable to a susceptibility artifact as the frequency band such as the IMF1 was covered by a respiratory frequency interval from 0.1 to $0.5 \mathrm{~Hz}$ (Cordes et al. 2001). However, several other studies detected that the spectral range of BOLD signals was greater than $0.1 \mathrm{~Hz}$, which also demonstrated consistent patterns with low-frequency fluctuations $(<$ $0.1 \mathrm{~Hz}$ ) (Boubela et al. 2013; Van Someren 2011). Furthermore, the orbitofrontal cortex detected in IMF1 might be the key region in DPD (Choe et al. 2013; Gottlich et al. 2013). Hence, we speculated that the high frequency band IMF1 might be associated with some physiological significance, rather than susceptibility artifact.

Meanwhile, these nodes with significant group effects were dominated in distinct frequency bands. From one perspective, it has been suggested that the brain oscillations within distinct frequency bands were generated by diverse mechanisms and possessed different physiological functions (Buzsaki and Draguhn 2004; Engel et al. 2001; Penttonen and Buzsáki 2003). In addition, the previous study (Oswal et al. 2013) suggested the associations between the distinct functional circuits oscillatory activities across multiple frequency bands and distinct motor, non-motor components of clinical impairment in Parkinson's disease. From another perspective, the distinct frequency characteristics of spatially distributed nodes were complicated, concerning unclarified origins, relations, and specific physiological functions of different oscillatory bands. Future work combining EEG, resting state fMRI as well as various task-based fMRI may contribute to a more precise understanding of the neurophysiological basis of the signals located in different frequency bands.

\section{Limitations}

In the current study, the comparison of frequency-specific brain networks was performed. Several limitations were noteworthy. The first limitation was due to the little-known influence of head motion on the frequency specificities in a small world network, since several recent studies (Power et al. 2012; Van Dijk et al. 2012) reported decreased long range connectivity and increased local connectivity due to head motion. Further investigation will be conducted in our next study. Moreover, other methods were not considered to define the value of FC, like partial correlation, which could estimate the direct interdependence after ruling out third-party effects (Pereda et al. 2005). Another limitation of our study was that the analysis of node definition was limited to AAL templatebased brain networks. A previous study suggested that the topological organization of brain networks was affected by various parcellation strategies (Wang et al. 2009). Future studies need to clarify the impact of various node definitions on this topic. Additionally, in the FC analysis in BOLD fMRI, an ongoing debate on the necessity to correct global signals in fMRI time courses did not reach a consensus (Hayasaka 2013). One previous study (Hayasaka 2013) suggested that without global signal correction, nodes along the interhemispheric fissure were highly connected while some nodes and subgraphs around white-matter tracts became disconnected from the rest of the network. In the current study, regression of global signals was performed. The discussion on how this preprocessing step influenced the results was beyond our focus. Lastly, the conclusion of the current study was demonstrated using limited sample data; further studies need to be done in a large cohort.

\section{Conclusion}

In the present study, we have introduced a novel method CEEMD to divide the resting state fMRI signals into five specific brain oscillations within distinct frequency bands. Our results demonstrated that brain networks for all groups showed small world architecture, and the optimal topological organization of brain networks decreased in both NDPD and DPD patients. Moreover, disrupted global topological organization, Lp, was distinct from frequency between NDPD and DPD groups. Additionally, altered brain regions within three key neural networks in NDPD and DPD patients and distinct frequency characteristics of these spatially distributed nodes were demonstrated. Overall, the present study demonstrated that there was dissociation in topological organizations of brain networks between NDPD and DPD patients in spatial and frequency domains. More importantly, the initial method described in the current study provided a novel insight to investigate the neuroimaging biomarkers of various brain disorders in both spatial and spectral domains.

Acknowledgments This work was supported by the National Natural Science Foundation of China under grant numbers: 81470816, 61131003 and 81271549.

\section{Compliance with Ethical Standards}

Funding This study was funded by the National Natural Science Foundation of China under grant numbers: 81470816, 61131003 and 81271549 .

Conflict of Interest Long Qian, Yi Zhang, Li Zheng, Xuemei Fu, Weiguo Liu, Yuqing Shang, Yaoyu Zhang, Yuanyuan Xu, Yijun Liu, Huaiqiu Zhu and Jia-Hong Gao declare that they have no conflict of interest.

Ethical approval All procedures performed in studies involving human participants were in accordance with the ethical standards of the institutional and/or national research committee and with the 1964 Helsinki declaration and its later amendments or comparable ethical standards. 
Open Access This article is distributed under the terms of the Creative Commons Attribution 4.0 International License (http:// creativecommons.org/licenses/by/4.0/), which permits unrestricted use, distribution, and reproduction in any medium, provided you give appropriate credit to the original author(s) and the source, provide a link to the Creative Commons license, and indicate if changes were made.

\section{References}

Aarsland, D., Pahlhagen, S., Ballard, C. G., Ehrt, U., \& Svenningsson, P. (2012). Depression in Parkinson disease-epidemiology, mechanisms and management. Nature Reviews. Neurology, 8(1), 35-47. doi:10.1038/nrneurol.2011.189.

Achard, S., Salvador, R., Whitcher, B., Suckling, J., \& Bullmore, E. (2006). A resilient, low-frequency, small-world human brain functional network with highly connected association cortical hubs. The Journal of Neuroscience, 26(1), 63-72. doi:10.1523/jneurosci. 3874-05.2006.

Altmann, L. J., \& Troche, M. S. (2011). High-level language production in Parkinson's disease: a review. Parkinsons Dis, 2011, 238956. doi: 10.4061/2011/238956.

Baggio, H. C., Sala-Llonch, R., Segura, B., Marti, M. J., Valldeoriola, F., Compta, Y., et al. (2014). Functional brain networks and cognitive deficits in Parkinson's disease. Human Brain Mapping. doi:10.1002/ hbm.22499.

Bai, F., Shu, N., Yuan, Y., Shi, Y., Yu, H., Wu, D., et al. (2012). Topologically convergent and divergent structural connectivity patterns between patients with remitted geriatric depression and amnestic mild cognitive impairment. The Journal of Neuroscience, 32(12), 4307-4318. doi:10.1523/jneurosci.5061-11.2012.

Barnes, J., \& David, A. S. (2001). Visual hallucinations in Parkinson's disease: a review and phenomenological survey. Journal of Neurology, Neurosurgery, and Psychiatry, 70(6), 727-733.

Bodis-Wollner, I. G., \& Paulus, W. (1999). Visual and visual cognitive dysfunction in Parkinson's disease: spatial and chromatic vision. Advances in Neurology, 80, 383-388.

Boubela, R. N., Kalcher, K., Huf, W., Kronnerwetter, C., Filzmoser, P., \& Moser, E. (2013). Beyond Noise: Using Temporal ICA to Extract Meaningful Information from High-Frequency fMRI Signal Fluctuations during Rest. Frontiers in Human Neuroscience, 7 , 168. doi:10.3389/fnhum.2013.00168.

Brown, P. (2003). Oscillatory nature of human basal ganglia activity: relationship to the pathophysiology of Parkinson's disease. Movement Disorders, 18(4), 357-363. doi:10.1002/mds.10358.

Bullmore, E., \& Sporns, O. (2009). Complex brain networks: graph theoretical analysis of structural and functional systems. Nature Reviews Neuroscience, 10(3), 186-198.

Bullmore, E. T., \& Bassett, D. S. (2011). Brain graphs: graphical models of the human brain connectome. Annual Review of Clinical Psychology, 7, 113-140.

Buzsaki, G., \& Draguhn, A. (2004). Neuronal oscillations in cortical networks. Science, 304(5679), 1926-1929. doi:10.1126/science. 1099745

Cardoso, E. F., Fregni, F., Maia, F. M., Melo, L. M., Sato, J. R., Cruz Jr., A. C., et al. (2010). Abnormal visual activation in Parkinson's disease patients. Movement Disorders, 25(11), 1590-1596. doi:10. 1002/mds.23101.

Choe, I. H., Yeo, S., Chung, K. C., Kim, S. H., \& Lim, S. (2013). Decreased and increased cerebral regional homogeneity in early Parkinson's disease. Brain Research, 1527, 230-237. doi:10.1016/ j.brainres.2013.06.027

Connolly, C. G., Wu, J., Ho, T. C., Hoeft, F., Wolkowitz, O., Eisendrath, S., et al. (2013). Resting-state functional connectivity of subgenual anterior cingulate cortex in depressed adolescents. Biological Psychiatry, 74(12), 898-907. doi:10.1016/j.biopsych.2013.05.036.

Cordes, D., Haughton, V. M., Arfanakis, K., Carew, J. D., Turski, P. A., Moritz, C. H., et al. (2001). Frequencies contributing to functional connectivity in the cerebral cortex in "resting-state" data. American Journal of Neuroradiology, 22(7), 1326-1333.

Engel, A. K., Fries, P., \& Singer, W. (2001). Dynamic predictions: oscillations and synchrony in top-down processing. Nature Reviews. Neuroscience, 2(10), 704-716. doi:10.1038/35094565.

Folstein, M. F., Folstein, S. E., \& McHugh, P. R. (1975). "Mini-mental state": a practical method for grading the cognitive state of patients for the clinician. Journal of Psychiatric Research, 12(3), 189-198.

Frodl, T., Bokde, A. L., Scheuerecker, J., Lisiecka, D., Schoepf, V., Hampel, H., et al. (2010). Functional connectivity bias of the orbitofrontal cortex in drug-free patients with major depression. Biological Psychiatry, 67(2), 161-167. doi:10.1016/j.biopsych. 2009.08.022

Gibb, W., \& Lees, A. (1988). The relevance of the Lewy body to the pathogenesis of idiopathic Parkinson's disease. Journal of Neurology, Neurosurgery \& Psychiatry, 51(6), 745-752.

Gottlich, M., Munte, T. F., Heldmann, M., Kasten, M., Hagenah, J., \& Kramer, U. M. (2013). Altered resting state brain networks in Parkinson's disease. PloS One, 8(10), e77336. doi:10.1371/journal. pone.0077336.

Hayasaka, S. (2013). Functional connectivity networks with and without global signal correction. Frontiers in Human Neuroscience, 7, 880.

He, Y., Chen, Z., \& Evans, A. (2008). Structural insights into aberrant topological patterns of large-scale cortical networks in Alzheimer's disease. The Journal of Neuroscience, 28(18), 4756-4766.

Hoehn, M. M., \& Yahr, M. D. (1998). Parkinsonism: onset, progression, and mortality. Neurology, 50(2), 318-318.

Hosseini, S. H., Hoeft, F., \& Kesler, S. R. (2012). GAT: a graphtheoretical analysis toolbox for analyzing between-group differences in large-scale structural and functional brain networks. PloS One, 7(7), e40709.

Huang, N. E., Shen, Z., Long, S. R., Wu, M. C., Shih, H. H., Zheng, Q., et al. (1998). The empirical mode decomposition and the Hilbert spectrum for nonlinear and non-stationary time series analysis. Proceedings of the Royal Society of London, Series A: Mathematical, Physical and Engineering Sciences, 454(1971), 903-995.

Huebl, J., Schoenecker, T., Siegert, S., Brucke, C., Schneider, G. H., Kupsch, A., et al. (2011). Modulation of subthalamic alpha activity to emotional stimuli correlates with depressive symptoms in Parkinson's disease. Movement Disorders, 26(3), 477-483. doi:10. $1002 /$ mds. 23515.

Itahashi, T., Yamada, T., Watanabe, H., Nakamura, M., Jimbo, D., Shioda, S., et al. (2014). Altered network topologies and hub organization in adults with autism: a resting-state FMRI study. PloS One, 9(4), e94115, doi:10.1371/journal.pone.0094115.

Jiao, Q., Ding, J., Lu, G., Su, L., Zhang, Z., Wang, Z., et al. (2011). Increased activity imbalance in fronto-subcortical circuits in adolescents with major depression. PloS One, 6(9), e25159. doi:10.1371/ journal.pone.0025159.

Joutsa, J., Rinne, J. O., Eskola, O., \& Kaasinen, V. (2013). Reduced striatal dopamine synthesis capacity is associated with symptoms of depression in patients with de novo unmedicated Parkinson's disease. J Parkinsons Dis, 3(3), 325-329. doi:10.3233/jpd-130205.

Kudlicka, A., Clare, L., \& Hindle, J. V. (2011). Executive functions in Parkinson's disease: systematic review and meta-analysis. Movement Disorders, 26(13), 2305-2315. doi:10.1002/mds.23868.

Kwak, Y., Peltier, S., Bohnen, N. I., Muller, M. L., Dayalu, P., \& Seidler, R. D. (2010). Altered resting state cortico-striatal connectivity in mild to moderate stage Parkinson's disease. Frontiers in Systems Neuroscience, 4, 143. doi:10.3389/fnsys.2010.00143. 
Laatu, S., Revonsuo, A., Pihko, L., Portin, R., \& Rinne, J. O. (2004). Visual object recognition deficits in early Parkinson's disease. Parkinsonism \& Related Disorders, 10(4), 227-233. doi:10.1016/ j.parkreldis.2004.02.001.

Leentjens, A. F., Verhey, F. R., Lousberg, R., Spitsbergen, H., \& Wilmink, F. W. (2000). The validity of the Hamilton and Montgomery-Åsberg depression rating scales as screening and diagnostic tools for depression in Parkinson's disease. International Journal of Geriatric Psychiatry, 15(7), 644-649.

Li, Q., Ke, Y., Chan, D. C., Qian, Z. M., Yung, K. K., Ko, H., et al. (2012). Therapeutic deep brain stimulation in Parkinsonian rats directly influences motor cortex. Neuron, 76(5), 1030-1041. doi:10.1016/j. neuron.2012.09.032.

Lin, C.-F., \& Zhu, J.-D. (2012). Hilbert-Huang transformation-based time-frequency analysis methods in biomedical signal applications. Proceedings of the Institution of Mechanical Engineers, Part H: Journal of Engineering in Medicine, 226(3), 208-216.

Liu, Y., Duan, Y., He, Y., Wang, J., Xia, M., Yu, C., et al. (2012). Altered topological organization of white matter structural networks in patients with neuromyelitis optica. PloS One, 7(11), e48846. doi:10. 1371/journal.pone.0048846.

Liu, Y., Liang, M., Zhou, Y., He, Y., Hao, Y., Song, M., et al. (2008). Disrupted small-world networks in schizophrenia. Brain, 131(4), 945-961.

Lui, S., Wu, Q., Qiu, L., Yang, X., Kuang, W., Chan, R. C., et al. (2011). Resting-state functional connectivity in treatment-resistant depression. The American Journal of Psychiatry, 168(6), 642-648. doi:10. 1176/appi.ajp.2010.10101419.

Luo, C., Chen, Q., Song, W., Chen, K., Guo, X., Yang, J., et al. (2014). Resting-state fMRI study on drug-naive patients with Parkinson's disease and with depression. Journal of Neurology, Neurosurgery, and Psychiatry, 85(6), 675-683. doi:10.1136/jnnp-2013-306237.

Marchand, W. R., Lee, J. N., Johnson, S., Thatcher, J., Gale, P., Wood, N., et al. (2012). Striatal and cortical midline circuits in major depression: implications for suicide and symptom expression. Progress in Neuro-Psychopharmacology \& Biological Psychiatry, 36(2), 290 299. doi:10.1016/j.pnpbp.2011.10.016.

Maslov, S., \& Sneppen, K. (2002). Specificity and stability in topology of protein networks. Science, 296(5569), 910-913.

McDonald, W. A., Richard, I. H., \& DeLong, M. R. (2003). Prevalence, etiology, and treatment of depression in Parkinson's disease. [Article; Proceedings Paper]. Biological Psychiatry, 54(3), 363375. doi:10.1016/s0006-3223(03)00530-4.

Mindham, R. H. (2001). Visual hallucinations in Parkinson's disease: their nature, frequency, and origins. Journal of Neurology, Neurosurgery, and Psychiatry, 70(6), 719-720.

Murray, L. L., \& Rutledge, S. (2014). Reading comprehension in Parkinson's disease. American Journal of Speech-Language Pathology, 23(2), S246-S258. doi:10.1044/2014_ajslp-13-0087.

Neufeld, M. Y., Blumen, S., Aitkin, I., Parmet, Y., \& Korczyn, A. D. (1994). EEG frequency analysis in demented and nondemented parkinsonian patients. Dementia, 5(1), 23-28.

Nijhuis, E. H., van Walsum, A.-M. v. C., \& Norris, D. G. (2013). Topographic hub maps of the human structural neocortical network. PloS One, 8(6), e65511.

Oswal, A., Brown, P., \& Litvak, V. (2013). Synchronized neural oscillations and the pathophysiology of Parkinson's disease. Current Opinion in Neurology, 26(6), 662-670. doi:10.1097/wco. 0000000000000034.

Penttonen, M., \& Buzsáki, G. (2003). Natural logarithmic relationship between brain oscillators. Thalamus \& Related Systems, 2(2), 145 152

Pereda, E., Quiroga, R. Q., \& Bhattacharya, J. (2005). Nonlinear multivariate analysis of neurophysiological signals. Progress in Neurobiology, 77(1), 1-37.
Postuma, R. B., Aarsland, D., Barone, P., Burn, D. J., Hawkes, C. H., Oertel, W., et al. (2012). Identifying prodromal Parkinson's disease: pre-motor disorders in Parkinson's disease. Movement Disorders, 27(5), 617-626. doi:10.1002/mds.24996.

Power, J. D., Barnes, K. A., Snyder, A. Z., Schlaggar, B. L., \& Petersen, S. E. (2012). Spurious but systematic correlations in functional connectivity MRI networks arise from subject motion. NeuroImage, 59(3), 2142-2154

Power, J. D., Cohen, A. L., Nelson, S. M., Wig, G. S., Barnes, K. A., Church, J. A., et al. (2011). Functional network organization of the human brain. Neuron, 72(4), 665-678.

Qian, L., Zhang, Y., Zheng, L., Shang, Y., Gao, J. H., \& Liu, Y. (2015). Frequency dependent topological patterns of resting-state brain networks. PloS One, 10(4), e0124681. doi:10.1371/journal.pone.0124681.

Ravina, B., Camicioli, R., Como, P. G., Marsh, L., Jankovic, J., Weintraub, D., et al. (2007). The impact of depressive symptoms in early Parkinson disease. [Article]. Neurology, 69(4), 342-347. doi:10.1212/01.wnl.0000268695.63392.10.

Rubinov, M., \& Sporns, O. (2010). Complex network measures of brain connectivity: uses and interpretations. NeuroImage, 52(3), 1059 1069.

Schrag, A., Barone, P., Brown, R. G., Leentjens, A. F., McDonald, W. M., Starkstein, S., et al. (2007). Depression rating scales in Parkinson's disease: critique and recommendations. Movement Disorders, 22(8), 1077-1092.

Sharman, M., Valabregue, R., Perlbarg, V., Marrakchi-Kacem, L., Vidailhet, M., Benali, H., et al. (2013). Parkinson's disease patients show reduced cortical-subcortical sensorimotor connectivity. Movement Disorders, 28(4), 447-454. doi:10.1002/mds.25255.

Shirer, W. R., Ryali, S., Rykhlevskaia, E., Menon, V., \& Greicius, M. D. (2012). Decoding subject-driven cognitive states with whole-brain connectivity patterns. Cerebral Cortex, 22(1), 158-165. doi:10. 1093/cercor/bhr099.

Sinanovic, O., Kapidzic, A., Kovacevic, L., Hudic, J., \& Smajlovic, D. (2005). EEG frequency and cognitive dysfunction in patients with Parkinson's disease. Medicinski Arhiv, 59(5), 286-287.

Skidmore, F., Korenkevych, D., Liu, Y., He, G., Bullmore, E., \& Pardalos, P. M. (2011). Connectivity brain networks based on wavelet correlation analysis in Parkinson fMRI data. Neuroscience Letters, 499(1), 47-51. doi:10.1016/j.neulet.2011.05.030.

Skidmore, F. M., Yang, M., Baxter, L., von Deneen, K., Collingwood, J., He, G., et al. (2013). Apathy, depression, and motor symptoms have distinct and separable resting activity patterns in idiopathic Parkinson disease. NeuroImage, 81, 484-495. doi:10.1016/j. neuroimage.2011.07.012.

Soikkeli, R., Partanen, J., Soininen, H., Paakkonen, A., \& Riekkinen Sr., P. (1991). Slowing of EEG in Parkinson's disease. Electroencephalography and Clinical Neurophysiology, 79(3), $159-165$.

Song, X., Zhang, Y., \& Liu, Y. (2014). Frequency Specificity of Regional Homogeneity in the Resting-State Human Brain. PloS One, 9(1), e86818.

Stam, C., Jones, B., Nolte, G., Breakspear, M., \& Scheltens, P. (2007). Small-world networks and functional connectivity in Alzheimer's disease. Cerebral Cortex, 17(1), 92-99.

Stebbins, G. T., \& Goetz, C. G. (1998). Factor structure of the Unified Parkinson's Disease Rating Scale: motor examination section. Movement Disorders, 13(4), 633-636.

Tomasi, D., \& Volkow, N. D. (2011). Functional connectivity hubs in the human brain. NeuroImage, 57(3), 908-917.

Trick, G. L., Kaskie, B., \& Steinman, S. B. (1994). Visual impairment in Parkinson's disease: deficits in orientation and motion discrimination. Optometry and Vision Science, 71(4), 242-245.

Van Den Heuvel, M. P., \& Hulshoff Pol, H. E. (2010). Exploring the brain network: a review on resting-state fMRI functional connectivity. European Neuropsychopharmacology, 20(8), 519-534. 
van den Heuvel, M. P., Stam, C. J., Kahn, R. S., \& Pol, H. E. H. (2009). Efficiency of functional brain networks and intellectual performance. The Journal of Neuroscience, 29(23), 7619-7624.

Van Dijk, K. R., Sabuncu, M. R., \& Buckner, R. L. (2012). The influence of head motion on intrinsic functional connectivity MRI. NeuroImage, 59(1), 431-438.

Van Someren, E. (2011). Spectral characteristics of resting state networks. Slow Brain Oscillations of Sleep, Resting State and Vigilance: Proceedings of the 26th International Summer School of Brain Research, Held at the Royal Netherlands Academy of Arts and Sciences, Amsterdam, The Netherlands, 29 June-2 July, 2010, 193, 259.

Vassar, S. D., Bordelon, Y. M., Hays, R. D., Diaz, N., Rausch, R., Mao, C., et al. (2012). Confirmatory factor analysis of the motor unified Parkinson's disease rating scale. Parkinson's disease, 2012, 719167.

Wang, J., Wang, L., Zang, Y., Yang, H., Tang, H., Gong, Q., et al. (2009). Parcellation-dependent small-world brain functional networks: a resting-state fMRI study. Human Brain Mapping, 30(5), 15111523. doi:10.1002/hbm.20623.
Watts, D. J., \& Strogatz, S. H. (1998). Collective dynamics of 'smallworld'networks. Nature, 393(6684), 440-442.

Wu, Z., \& Huang, N. E. (2009). Ensemble empirical mode decomposition: a noise-assisted data analysis method. Advances in Adaptive Data Analysis, 1(01), 1-41.

Xie, H., \& Wang, Z. (2006). Mean frequency derived via Hilbert-Huang transform with application to fatigue EMG signal analysis. Computer Methods and Programs in Biomedicine, 82(2), 114-120.

Yeh, J.-R., Shieh, J.-S., \& Huang, N. E. (2010). Complementary ensemble empirical mode decomposition: A novel noise enhanced data analysis method. Advances in Adaptive Data Analysis, 2(02), 135156.

Yu, R., Liu, B., Wang, L., Chen, J., \& Liu, X. (2013). Enhanced functional connectivity between putamen and supplementary motor area in Parkinson's disease patients. PloS One, 8(3), e59717. doi:10. 1371/journal.pone.0059717.

Zhang, Z., Liao, W., Chen, H., Mantini, D., Ding, J. R., Xu, Q., et al. (2011). Altered functional-structural coupling of large-scale brain networks in idiopathic generalized epilepsy. Brain, 134(Pt 10), 2912-2928. doi:10.1093/brain/awr223. 